\title{
O.S.P.
}

L'orientation scolaire et professionnelle

$36 / 1 \mid 2007$

Insertion, biographisation, éducation

\section{La question de l'expertise dans le conseil en orientation avec les personnes handicapées}

Vocational counseling for individuals with disabilities: An expert advice?

Joëlle Mezza

\section{OpenEdition}

Journals

Édition électronique

URL : https://journals.openedition.org/osp/1328

DOI : $10.4000 /$ osp. 1328

ISSN : 2104-3795

Éditeur

Institut national d'étude du travail et d'orientation professionnelle (INETOP)

Édition imprimée

Date de publication : 15 mars 2007

Pagination : 96-105

ISSN : 0249-6739

\section{Référence électronique}

Joëlle Mezza, «La question de l'expertise dans le conseil en orientation avec les personnes

handicapées ", L'orientation scolaire et professionnelle [En ligne], 36/1 | 2007, mis en ligne le 05 mars

2010, consulté le 18 janvier 2022. URL : http://journals.openedition.org/osp/1328 ; DOI : https://

doi.org/10.4000/osp.1328

Ce document a été généré automatiquement le 18 janvier 2022.

(c) Tous droits réservés 


\title{
La question de l'expertise dans le conseil en orientation avec les personnes handicapées ${ }^{1}$
}

\author{
Vocational counseling for individuals with disabilities: An expert advice?
}

Joëlle Mezza

\section{Introduction}

1 L'altération de l'intégrité physique ou mentale de nos semblables ne nous laisse pas indifférents et nous touche même souvent profondément. Le handicap nous concerne dans l'organisation même de notre sensibilité, comme si nous pouvions risquer un jour de devenir nous-mêmes sourds, paralysés ou fous. La déficience nous affecte intimement dans l'image inconsciente de notre corps et dans la mémoire de nos difficultés passées. Elle nous interpelle sans que nous puissions nous en défendre. Elle suscite de l'intérêt, de la gêne ou de l'angoisse. Ces émotions sont à l'origine de réactions de protection, d'un désir de réparation, d'un déni du handicap ou autre. Elles constituent un phénomène naturel qui affecte l'imaginaire humain dans son ensemble.

2 Les professionnels de l'orientation, en contact avec des personnes handicapées, sont eux aussi confrontés à de telles émotions. Celles-ci risquent d'influer sur leurs pratiques en dehors de tout contrôle conscient. Pour ces professionnels, il importe donc, avant tout, de prendre conscience de ces affects, afin d'éviter qu'ils n'agissent en sous-main et ne déterminent leurs activités à leur insu. Ringler (2004) souligne ainsi : «Ce n'est qu'en prenant acte de nos réactions affectives inconscientes que l'on parvient à adopter une attitude juste et naturelle avec les enfants handicapés » (p. 108).

3 En tout état de cause, les représentations que les professionnels de l'orientation se font $\mathrm{du}$ handicap ne peuvent manquer d'avoir un impact sur leurs pratiques professionnelles. Mais existe-t-il, en matière de conseil en orientation, des pratiques spécifiques souhaitables quand on travaille avec un public de personnes handicapées? 
Quels sont les points communs avec le travail face à un public ordinaire ? Jusqu'à quel point l'activité de conseil est-elle la même ? Jusqu'à quel point est-elle différente ?

4 C'est en se fondant sur l'expérience concrète que nous avons de la réception des personnes handicapées au Centre d'information et d'orientation (CIO) spécialisé de Paris que nous tenterons d'apporter des éléments de réponse à ces questions.

\section{Des demandes convergentes adressées aux conseillers d'orientation psychologues du $\mathrm{CIO}$ spécialisé : être des experts utilisant des tests}

5 Notre point de départ sera celui des représentations courantes que les conseillers d'orientation psychologues exerçant dans des CIO accueillant un public tout venant semblent se faire de l'activité de conseil au CIO spécialisé. Lors de leurs appels téléphoniques à ce $\mathrm{CIO}$, plusieurs thèmes apparaissent en effet de manière récurrente : ce dernier disposerait d'une documentation et d'outils spécifiques et l'on y utiliserait plus qu'ailleurs des tests psychotechniques. L'idée implicite serait donc que les conseillers de ce $\mathrm{CIO}$ seraient - notamment lors de leur activité d'aide à l'élaboration d'un projet de vie - davantage dans une posture d'expert que les conseillers des autres CIO. Pour le dire rapidement : ils auraient des outils pour savoir, et même, pour « savoir à la place de l'autre ».

6 Une telle représentation trouve vraisemblablement une part de son origine dans le modèle médical qui a longtemps été dominant dans l'approche du handicap. Mais elle renvoie sans doute aussi aux représentations et affects (évoqués ci-dessus) que suscite le handicap et à la crainte corrélative d'être incapable - en tant que conseiller - d'y faire face. C'est-à-dire, fondamentalement, à la peur d'être un professionnel incompétent : l'idée de disposer d'outils ad hoc, le rêve d'avoir des méthodologies et des instruments spécifiques peuvent être compris comme des produits de ces craintes.

Cette inquiétude se manifeste aussi dans la première réaction qu'expriment certains conseillers d'orientation psychologues qui, seulement habitués à un public tout venant, se trouvent devoir accompagner un jeune en situation de handicap. Dans bien des cas, ils déclarent alors immédiatement - « je vais lui faire passer des tests!»-sans avoir analysé au préalable la situation réelle du jeune en question. Pourquoi, en effet, un élève myopathe scolarisé en classe de quatrième ordinaire et sans retard scolaire devrait-il subir un examen de son potentiel intellectuel, pour la seule raison qu'il souhaite intégrer une "unité pédagogique d'intégration handicap moteur », du fait de l'évolution de sa maladie? Quelle est la pertinence de "faire passer une Noria » à un jeune déficient visuel ayant obtenu son brevet des collèges, mais dont il faut envisager la réorientation vers une autre filière professionnelle, après quelques semaines de scolarisation dans un BEP inadapté?

Des demandes d'expertises - impliquant plus ou moins implicitement l'usage de tests sont aussi régulièrement adressées au CIO spécialisé par des instances administratives, telles que la Commission départementale d'orientation vers les enseignements adaptés du second degré (CDOEA) ou la Commission des droits et de l'autonomie des personnes handicapées (CDAPH). Ces commissions doivent trancher en termes de solutions scolaires, face à des dossiers souvent délicats. Leurs demandes - souvent formulées de 
façon globale-utilisent des vocables variés: tests, bilan, évaluation, examen psychologique, etc.

9 Des souhaits semblables sont parfois exprimés par des parents. Souvent, le scénario est le suivant : ou bien ces parents sont en désaccord avec les décisions d'orientation de l'administration scolaire, ou bien ils se trouvent démunis face à un échec scolaire durable ou aux difficultés d'insertion professionnelle de leur enfant. Pour eux - c'est ainsi qu'ils formulent généralement les choses - il s'agit d'y voir plus clair quant au potentiel réel de ce dernier : l'usage de tests devrait permettre de le révéler.

\section{La place des tests dans les interventions de conseil en orientation auprès des jeunes porteurs d'un handicap}

10 Confronté à de telles demandes convergentes, le conseiller d'orientation psychologue, dont la mission relative aux personnes handicapées est - selon les termes de la loi du 11 février 2005 - de les aider à "formuler un projet de vie", ne peut manquer de s'interroger sur sa posture. Est-il plutôt un expert qui évalue et décrit en vérité les atouts, les faiblesses et les potentialités d'un consultant qu'il observe de l'extérieur? Est-il plutôt un accompagnateur qui aide la personne à s'engager dans un processus réflexif de construction de soi mettant en perspective la diversité de ses expériences passées, présentes et anticipées?

11 C'est plutôt l'idéal que définit cette seconde question qui guide les conseillers d'orientation psychologues du CIO spécialisé de Paris. Pour eux, il s'agit avant tout d'accompagner le jeune dans la formulation de son projet de vie. C'est donc dans cette perspective fondamentale que le test peut trouver sa place. Très concrètement, cela signifie d'abord que - plus encore que pour un public tout venant - la question de l'opportunité du test est très sérieusement examinée : en vue de quoi le test sera-t-il utilisé? (Quelle sera sa finalité? Aider la personne à repérer certaines de ses potentialités? Jeter un regard différent sur son expérience? Fournir des «données objectives " à une commission pour l'aider à orienter une personne? Etc.) Quelles informations souhaite-t-on obtenir? Quel outil serait le plus pertinent ou le mieux adapté dans ce contexte?

12 Cette réflexion préalable à l'usage du test et de ses modes d'intervention se trouve au cœur même de la déontologie du psychologue, de ses droits et de ses devoirs. Une telle réflexion semble d'une importance d'autant plus grande, s'agissant des jeunes handicapés que, comme on l'a vu, d'une part, les demandes convergent de mettre en œuvre avec eux de tels outils et que, d'autre part, ceux-ci peuvent venir combler l'inquiétude du conseiller (ou encore le protéger face aux demandes pressantes mais non distanciées de l'administration ou des familles). Comme le note Bourgès (1975), de tels outils visent en effet souvent à combler «un besoin inassumable de réassurance » (p. 9).

13 Aux États-Unis, il existe différents outils, méthodes et tests adaptés aux personnes handicapées (voir Zunker, 2002, pp. 382-400). Par exemple, Micro-Tower (acronyme pour Testing, Orientation, and Work Evaluation in Rehabilitation) comprend des tests portant sur des échantillons de travail. Ils visent à mesurer 5 aptitudes primaires : motrice, perspicacité dans le travail administratif, spatiale, numérique, verbale. Les échantillons de travail sont notamment: de l'assemblage électronique, de l'emballage, 
du codage de courrier, de la vérification de dossiers, de la lecture de plans, du rendu de monnaie à une caisse, de la prise de messages, etc. Il existe aussi des tests destinés à des élèves déficients mentaux. Ainsi, la batterie d'informations sociales préalables à l'orientation est destinée à des collégiens et lycéens. Elle porte sur 9 domaines renvoyant aux 5 objectifs fondamentaux des programmes scolaires qui leur sont destinés : l'employabilité, l'autosuffisance économique, la vie de famille, les habitudes personnelles et les habiletés à communiquer. Par exemple, s'agissant de l'autosuffisance économique, le test porte sur les habitudes d'achat, l'établissement d'un budget et la tenue de son compte bancaire.

En France de tels outils sont quasi-inexistants et le CIO spécialisé de Paris ne dispose d'aucun matériel spécifique en matière d'évaluation psychotechnique. Des impossibilités matérielles surgissent donc : comment faire passer une épreuve visuelle à un aveugle ou un amblyope ? Comment faire passer et évaluer une épreuve graphique ou demandant des manipulations à quelqu'un souffrant de problèmes de motricité ? Comment faire passer des épreuves verbales à quelqu'un qui ne parle pas ? Comment interpréter une lenteur d'exécution, caractéristique transversale à de nombreux handicaps?

L'interprétation des tests est toujours une affaire délicate. Elle l'est encore plus avec les personnes handicapées car elle est loin de lever toutes les incertitudes. Par exemple : dans la réussite ou l'échec à un test, comment déterminer ce qui tient à une impossibilité due au handicap physique ou sensoriel et ce qui tient à une certaine déficience intellectuelle ? Bourgès (1975) rappelle que tout test fait appel à trois types de facteurs étroitement intriqués: des facteurs intellectuels qui supposent la compréhension d'une consigne et la coordination mentale entraînant une réponse, des facteurs instrumentaux induits par la participation plus ou moins importante du corps dans la réponse et, enfin, des facteurs affectifs. Alors : quel sens donné à un score échoué ? Comment faire la part des choses? Qu'est-ce qui relève, par exemple, d'une déficience intellectuelle profonde, de l'histoire personnelle de l'individu ou encore du contexte actuel? Par ailleurs, s'il peut être riche d'enseignement de situer une personne par rapport à une population de référence, il arrive que certaines personnes lourdement handicapées ne présentent qu'un tableau de déficiences par rapport à un étalonnage établi sur une population générale. Quels enseignements le conseiller d'orientation psychologue peut-il tirer de ces observations? Comment faire pour les exploiter?

C'est en évoquant le cas de Christelle que nous tenterons d'apporter des éléments de réponse à ces questions.

\section{Accompagner Christelle - et sa famille - dans son orientation}

Lorsqu'elle est reçue pour la première fois au CIO spécialisé, Christelle est âgée de 13 ans. Elle présente un handicap moteur très important, que son père qualifie de "retard psychomoteur sans étiologie», le corps médical n'ayant pas trouvé d'explication. La jeune fille, en fauteuil roulant électrique, donne l'impression « d'une poupée de chiffon ». Elle souffre en outre d'un nystagmus, problème visuel mineur par 
rapport à la massivité de sa déficience motrice. Christelle est scolarisée à domicile depuis longtemps. Au moment de sa venue au CIO, elle suit les cours du CE2.

C'est à la demande de la Commission de circonscription du second degré (CCSD) et de sa famille que Christelle est reçue. L'objectif annoncé est de trouver une solution de scolarisation autre qu'à domicile. Christelle a en effet passé quelques mois l'année précédente à temps partiel dans une classe d'intégration scolaire (CLIS) « retard scolaire ». Mais cette expérience, qui s'est avérée très positive du point de vue de la socialisation de la jeune fille, n'a pu être prolongée à cause de l'âge trop avancé de Christelle. La demande de la CCSD est que le CIO spécialisé établisse un bilan de ses potentialités afin de proposer une solution scolaire la plus adaptée possible.

amille de Christelle - très en colère contre l'Éducation nationale, jusque là incapable à ses yeux de trouver une solution de scolarisation durable pour sa fille - a déjà refusé la proposition qui lui a été faite d'une classe de section d'enseignement général et professionnel adapté (SEGPA). De leur côté, les parents sont eux aussi très demandeurs d'une évaluation de leur enfant, afin d'avoir un regard plus objectif sur ce que pourrait leur proposer la CCSD.

Lors de sa première rencontre avec la conseillère d'orientation psychologue, Christelle est accompagnée de ses parents. Ceux-ci manifestent alors un comportement que la conseillère perçoit comme très défensif. Ils lui apparaissent comme ayant une attitude ambiguë par rapport au handicap de leur fille, semblant tantôt être tout à fait conscients de ses difficultés et tantôt nier le handicap en faisant l'inventaire de la multitude d'activités dans lesquelles elle s'investit (par exemple : elle apprend à jouer de la harpe). Un fait frappe la conseillère : en plus de son handicap moteur lourd, la jeune fille ne parle presque pas.

21 La question des tests à mettre en œuvre apparaissait particulièrement délicate : quels instruments utiliser avec une jeune fille qui ne peut pas parler et difficilement écrire? La conseillère, après s'être longuement concertée avec ses collègues du CIO, décida de lui proposer les épreuves suivantes :

- Le dessin de la dame de Fay qui permet d'observer le niveau d'élaboration des dessins et la capacité à écrire sous la dictée ou à copier une phrase.

- Les épreuves de performance du WISC III et B de la figure de Rey qui fournissent des éléments d'analyse des capacités de raisonnement logique (dans le cas de Christelle, l'usage des épreuves verbales du WISC III apparaissait exclu).

22 Au cours de l'interaction de conseil, la conseillère dut renoncer à la figure de Rey: le niveau $\mathrm{B}$ - pourtant destiné à des enfants à partir de 8 ans - s'avéra trop difficile pour Christelle qui ne put tracer le moindre trait. Le niveau d'élaboration du dessin, qu'elle a réalisé selon le protocole de la dame de Fay, correspondait, pour sa part, à celui d'un enfant de quatre à cinq ans d'âge mental. Par ailleurs, la jeune fille n'a pu recopier que quelques mots de la phrase courte illustrant le dessin, phrase que la conseillère se proposait au départ de lui dicter. Aux épreuves de performance du WISC III, Christelle obtint des résultats très homogènes, la situant à un âge mental inférieur à celui d'un enfant de six ans.

Dans un tel cas, comment procéder pour rendre compte à Christelle et à sa famille de tels résultats sans se limiter à dresser une liste de déficiences? CIO spécialisé. Ces concertations conduisirent à proposer que les parents de Christelle 
fussent d'abord reçus - sans leur fille - par la directrice du CIO spécialisé. Une telle démarche semblait en effet offrir de nombreux avantages. D'abord, une réception par la directrice du CIO témoignait de la reconnaissance qui leur était accordée. Ensuite, cette amorce de séparation d'avec leur enfant permettait que ces parents soient mieux pris en compte en tant que sujets : leur souffrance et leurs récriminations pouvaient être entendues. Enfin, ce dialogue pouvait donner une idée de la manière dont la famille faisait face à cette situation.

Le compte rendu des résultats aux tests n'est intervenu qu'ensuite: au cours de la quatrième rencontre entre la conseillère, Christelle et ses parents. La conseillère indiqua tout d'abord très clairement aux parents comment se situait la jeune fille par rapport à sa classe d'âge, c'est- à-dire en ne leur masquant pas le niveau de leur enfant (tel que les tests le manifestaient). La conseillère, par ailleurs, mit l'accent sur certaines des potentialités de Christelle que l'interaction de conseil avait permis de mettre à jour. En l'occurrence : des capacités d'apprentissage - liées en partie à son grand investissement dans les tâches - lui donnant un potentiel d'adaptation scolaire, des capacités de mémorisation, une capacité à reproduire des mécanismes, et, enfin, une ouverture certaine sur le monde extérieur(certes dans la limite de ses potentialités).

26 La relation de confiance qui avait pu être construite avec la famille a sans doute fortement contribué à ce que ce compte rendu et cet échange se passent dans de bonnes conditions: les parents étaient désormais en mesure de dialoguer avec la conseillère et d'accueillir certaines des propositions qu'elle pouvait faire à leur enfant.

L'année suivante, Christelle a été scolarisée à temps partiel au sein de l'unité pédagogique d'intégration (UPI) « handicap moteur » d'un collège de banlieue, dans le souci premier de développer sa socialisation et son autonomie. Les parents ont, par la suite, rappelé la conseillère pour lui donner des nouvelles de Christelle et lui dire qu'ils étaient heureux de cette solution.

Un cas comme celui de Christelle n'est pas exceptionnel. Il nous semble, de plus, exemplaire en ce qu'il révèle clairement que c'est seulement dans le cadre d'une démarche d'accompagnement que l'usage des tests et leurs résultats trouvent leur sens. S'agissant de Christelle, c'est à peine caricaturer que de dire que la demande institutionnelle était la suivante : dites-nous son niveau d'aptitude de manière à ce que l'on puisse la « caser » dans une structure ad hoc. Les parents résistaient à leur façon à cette demande qui suscitait chez eux la crainte d'une relégation de leur enfant: ils réclamaient des tests (qui, à leurs yeux, devaient sans doute montrer que Christelle avaient beaucoup plus de potentialités que l'institution scolaire ne l'imaginait). L'interaction de conseil a permis de lire dans une perspective différente la situation de Christelle. Au cours de celle-ci, la question centrale est en effet devenue pour chacun : comment permettre au mieux à Christelle de développer ses potentialités et de se construire en tant que personne dans la société où elle vit? Dans cette nouvelle perspective, les tests ont été perçus comme des outils susceptibles d'apporter des éléments d'information utiles - parmi d'autres informations - pour élaborer, ensemble, le meilleur scénario possible pour l'orientation du jeune concerné. 


\section{Outils, ressources et réseau pour développer des interventions adaptées} les brochures de la collection "Réadaptation » de l'Office national d'information sur les enseignements et les professions (ONISEP). Celles-ci recensent l'ensemble des établissements spécialisés en France en les classant par types de handicap. En effet, contrairement au discours dominant prônant le « tout intégration », les établissements spécialisés correspondent à une demande fréquente des familles. Pour plusieurs raisons. Dans certains cas, elles ont déjà été confrontées à des scolarisations en milieu ordinaire qui se sont avérées infructueuses. Dans d'autres, les adolescents arrivent à un moment de leur parcours scolaire où ils souhaitent être en milieu plus protégé. Enfin, parfois, la lourdeur du handicap est telle qu'elle ne permet pas d'envisager une scolarisation ordinaire.

Si les brochures de l'ONISEP constituent une aide indubitable pour les conseillers d'orientation psychologues du CIO spécialisé, il est une autre ressource extrêmement précieuse que celui-ci n'a pu développer que grâce à son existence même : il s'agit du réseau de partenaires qu'il s'est forgé au fil du temps. Ce réseau est constitué d'une multiplicité de personnes travaillant dans une grande variété d'institutions : référents handicap dans des centres de formation à l'apprentissage (CFA) ou en missions locales, chefs d'établissement scolaire, interlocuteurs des cellules relais-handicap des 
universités, directeurs des ressources humaines d'entreprises cherchant à intégrer des personnes handicapées, conseillers d'orientation psychologues des CIO tout venant, etc. Un tel réseau constitue un atout majeur à la fois pour les personnes handicapées et pour les conseillers d'orientation psychologues qui les conseillent. En effet, pouvoir adresser une personne dont on connaît la problématique personnelle à un interlocuteur privilégié facilite grandement son parcours et son intégration. Les échanges qui se développent avec ces partenaires sont fondamentaux. Ils se fondent sur la réciprocité et la confiance. Ce point est déterminant: l'expérience montre que l'élaboration - foncièrement interminable - d'un projet de vie par une personne handicapée s'inscrit dans la durée et qu'elle nécessite de nombreux allers- retours que de tels échanges entre partenaires permettent d'accompagner.

\section{Conclusion}

Quelle réponse apporter à notre question initiale ? Peut-on affirmer que les pratiques de conseil en orientation avec des personnes handicapées ont une spécificité ?

$\mathrm{Au}$ terme de ce rapide survol des pratiques du CIO spécialisé de Paris, il est difficile de répondre d'une manière tranchée à cette question. En effet, d'un côté, il est clair que les pratiques de conseil avec ces publics ont les mêmes finalités que celles destinées au public tout venant: il s'agit d'accompagner les jeunes dans leur construction de soi, notamment en les aidant à formuler des projets de vie (des projets personnels, de formation, professionnels) et en s'engageant activement dans leur réalisation. Cependant, d'un autre côté, la particularité de la situation des personnes handicapées conduit souvent à donner une inflexion particulière aux interactions de conseil qui sont développées avec elles. De nombreux facteurs jouent un rôle : la sidération que le conseiller peut ressentir face à un handicap, les demandes convergentes de mettre en œuvre des tests, la difficulté à trouver des outils d'observation qui soient adaptés à certaines personnes handicapées, la difficulté à échanger verbalement avec certaines d'entre elles, les souffrances, les attentes et, parfois la culpabilité des familles, la difficulté de certains jeunes handicapés à vivre leur propre situation, etc.

Tous ces facteurs conduisent les conseillers d'orientation psychologues du CIO spécialisé à devoir réfléchir collectivement - sans doute plus fréquemment que leurs collègues des $\mathrm{CIO}$ tout venant - sur leurs pratiques, sur les outils dont ils disposent, sur les usages qu'ils en font et sur le cas des personnes qu'ils rencontrent, etc. On peut postuler que l'engagement dans de telles réflexions et analyses se traduit à la fois par un enrichissement et une certaine homogénéisation de leurs pratiques. Ceci apparait nettement dans les cas où, détournant certains outils de leurs objectifs premiers, ils les réaménagent afin d'accompagner une personne qu'affecte tel ou tel handicap. Enfin, il est sans doute une expérience dont ces conseillers sont plus familiers que les autres: celle de la probabilité de la mort prochaine d'un de leur consultant. Quelle interaction de conseil engager, par exemple, avec un jeune myopathe dont la santé se dégrade de mois en mois? Que faire pour l'aider à faire face à son angoisse et pour soutenir son désir de vivre et de se projeter dans l'avenir? N'est-il pas alors raisonnable d'encourager, non sans inquiétude, des projets que tout "décideur rationnel» considérerait comme parfaitement utopiques? 


\section{BIBLIOGRAPHIE}

Bourgès, S. (1975). Approche génétique et psychanalytique de l'enfant : essai et synthèse du bilan psychologique. Vol. I : Choix et interprétation des épreuves. Paris : Librairie Riche.

Ringler, M. (2004). Comprendre l'enfant handicapé et sa famille (2éd.). Paris : Dunod.

Sontag, J.-C. (2002). L'Épreuve visuelle d'intérêts. Épreuve et manuel d'application. Paris : Éditions du Centre de psychologie appliquée.

Zunker, V. G. (2002). Career counselling : applied concepts of life planning (6 $6^{\mathrm{e}}$ é.). Pacific Grove, CA : Brooks/Cole.

\section{NOTES}

1. Cet article reprend les thèmes développés dans un exposé lors du séminaire du 3 mars 2006 «Quelles pratiques d'orientation avec les personnes en situation de handicap? " du laboratoire de psychologie de l'orientation de l'INETOP. Jean Guichard a participé à la mise au point de ce texte.

2. Les tests de la Noria ont été créés, à partir des années soixante-dix, par Yves Wendels, psychologue spécialisé dans l'évaluation. Il existe différentes épreuves de sondage scolaire en français et en mathématiques, le plus souvent destinées à des élèves présentant des difficultés d'apprentissage.

\section{RÉSUMÉS}

Existe-t-il des activités spécifiques de conseil en orientation avec un public de personnes handicapées? Une observation des pratiques développées par les conseillers d'orientation psychologues du CIO de Paris spécialisé dans l'accompagnement de ces publics met en évidence qu'une demande récurrente leur est adressée : celle d'être des experts utilisant des tests. Par ailleurs, la particularité (et aussi la difficulté) de la situation de certaines personnes handicapées conduit les conseillers d'orientation psychologues du CIO spécialisé à devoir réfléchir collectivement - sans doute plus fréquemment que les autres conseillers - sur leurs pratiques, sur les outils dont ils disposent, sur les usages qu'ils en font et sur le cas des personnes qu'ils rencontrent, etc. On peut postuler que l'engagement dans de telles réflexions et analyses se traduit à la fois par un enrichissement et une certaine homogénéisation de leurs méthodologies. La finalité de leurs pratiques apparaît néanmoins identique à celles destinées au public tout venant : accompagner les jeunes (et les adultes) dans leur construction de soi, notamment en les aidant à formuler des projets de vie (des projets personnels, de formation, professionnels) et en s'engageant activement dans leur réalisation.

Do specific vocational guidance activities exist for the orientation of disabled people? An observation of the practices developed by the psychological career advisers of the CIO in Paris, 
specialized in the accompaniment of this public, highlights that convergent requests are addressed to them : to be experts and to use tests. In addition, the characteristic situation (which is also the difficulty) of certain disabled people obliges the specialized psychological career advisers of the CIO to reflect collectively-undoubtedly more frequently than the other adviserson their practices, on their tools and on uses that they make of them, as well as on the cases of the people whom they meet, etc. One can postulate that engagement in such reflections and analyses, results both in enrichment and in a certain homogenization of their methodologies. Nevertheless, the finality of their practices appears identical to those intended for the general public: to accompany the young people (and the adults) in their self-construction, in particular by helping them to formulate life projects (personal projects, training projects, professional projects), while engaging actively in their realization.

\section{INDEX}

Mots-clés : Accompagnement, Conseil en orientation, Expertise, Personne handicapée, Test

Keywords : Counselling, Disabled person, Expert advice, Test, Vocational guidance

\section{AUTEUR}

\section{JOËLLE MEZZA}

Actuellement formatrice à l'INETOP (41, rue Gay Lussac, 75005 Paris), Joëlle Mezza fut précédemment conseillère d'orientation psychologue au Centre d'information et d'orientation de Paris spécialisé dans l'accueil des personnes handicapées. Courriel : joelle.mezza@cnam.fr 